
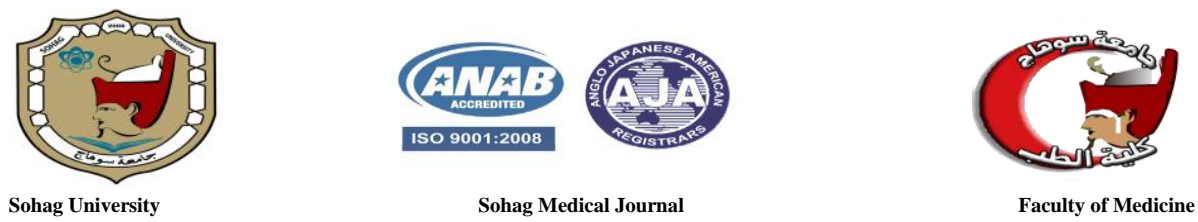

\title{
Effect of administration of lead acetate on the post natal development of ovary in albino rats
}

\author{
SALAH R. AHMED*, SALWA M. OUIES**, \\ ASMAA S. BASSIT** \\ *obesterics and gynacology faculty of medicine sohag university \\ ** anatomy department, faculty of medicin,sohage university, sohage
}

\begin{abstract}
:
Introduction: Exposure to lead is still a major medical dilemma in both environmental and occupational settings. The toxicity of lead, on adult female reproduction, results in reduced fertility and low pregnancy outcomes.

Aim of the work: This research aims to reveal the effect of lead acetate on the development of ovary on albino rats.

Materials and methods: In this study, a total of 20adult pregnant female albino rats were used. The animals were divided into two groups each consist of 10 rats: Group 1(control): take distilled water. Group 2(lead treated): take lead acetate in a dose of $(640 \mathrm{mg} / \mathrm{kg})$, were administrated orally on every day from gestational day 10 to postnatal day 21.then 10 female pups from each group were randomly selected, slaughter at age of one and two weak then ovary was taken and subjected to light microscopic study.

Result: lead exposure was dangerous, caused severe pathological changes in the ovarian follicle in the form of delayed development of primordial follicle, damage to granulosa cell and degeneration to the oocyte.

Conclusion: The administration to lead in the neonatal period affects the growth of primordial follicles during postnatal and juvenile life

Keywords: lead acetate,ovary,follicle development
\end{abstract}

\section{Introduction:}

The ovaries in the female rate are reproductive organs responsible for the production of estragon and progesterone. The Ovary formed of the ovarian follicle which is the important unit of the ovary. Follicles go through various stages of development, at Neonatal Period( Birth to PND 7) the primordial, the primary is apparent, the follicles predominated in the medullary and cortical regions, at Early infantile period (PND 8-14) the secondary follicles and tertiary follicle present at the central part of the ovary in addition to the primary follicle. (1). primordial follicles present at the time of birth and diminish progressively until they disappear at the time of the menopause. It consists of a primary oocyte surrounded by

one layer of the flattened cell. The primary follicle formed one layer of columnar cells surrounded oocyte. (2).

Secondary follicles formed of a multilayered zone of granulosa cells( the zona granulosa) surround the oocyte. 
SOHAG MEDICAL JOURNAL

Vol. 24 No. 3 July 2020

And a layer of glycoprotein coat called the zona pellucida, between the oocyte and the zona granulosa, tertiary follicle (antral follicles) it a form of cystic spaces coalesce and form a large central cavity called the follicular antrum and the granulosa cell surrounded by theca cell that divided into two zones: a theca interna and theca externa (3).

Lead is a metal that is widely distributed in the environment, the condition in which exposure is high can be found in manufacturing areas. (4).

lead can cross the umbilical cord from mother to fetus, precipitate in the fetus during pregnancy and can be passed to the fetus during lactation so it produced adverse effects during fetal development. (5)

There is a serious difference between species in their response to lead and the compound containing lead. The toxicity also varies between the chemical form of the lead. Lead acetate is very soluble and more dangerous than insoluble lead oxides (6).

Oral intake of lead in large doses leads to a decrease in the number of ovarian follicles indicate a high relationship between the ratio of lead in circulation and atresia of the follicles of rate, also lead exposure may change steroid production and gonado-trophin binding in the ovaries of adult rats(7).

\section{MATERIALS AND METHODS:}

In this study, a total of 20adult pregnant female albino rats their weight range from 200-250g were used. The animals were brought from the animal house of the Assiut faculty of medicine. They were reared under-the standard conditions of feeding, light-dark ratio, and temperature, in sohage faculty of medicine animal house.
Effect of administration of lead acetate

ASMAA S. BASSIT.et al
The rat was kept in plastic cages in the ratio of one per four males and females. Females were detected to be pregnant by the presence of vaginal plugs (an indication of the pres-ence of sperm in the vagina) and separated for the experimental protocol and their gestational days were recorded.

The animals were divided into two groups:

I-Group 1(control): Includes 10 female offspring of 10 mothers who received distilled water.

II-Group 2(lead treated): Includes 10 female offspring's of 10 mothers received lead acetate in a dose of (640mg/kg), ( 8) were administrated orally on every day from gestational day 10 to postnatal day 21 .

\section{Drug, dosage, and administration:}

Lead acetate was brought from Alex bioscience in the form of powder $10 \mathrm{gm}$ of lead dissolve in $100 \mathrm{~cm}$ of saline.

\section{Methods:}

The female offspring from each of the groups were randomly selected, slaughter at age of one weak and two weak, by intramuscular IM injection of a mixture of Ketamine $(90 \mathrm{mg} / \mathrm{kg}$ body weight) and Xylazine (10 mg/kg body weight) then their ovaries were taken for light microscope study. The section fixed in $10 \%$ natural buffered formalin and processed for light microscopic study to get a paraffin section of 6um thickness, the sections stained with Haematoxylin and Eosin.

Morphometric and statistical analysis: Estimation of the diameter of the ovary done by using (digimizer version 3.7.2005-2010) Medcale software in the anatomy depar-tment at sohage univer- 
SOHAG MEDICAL JOURNAL

Vol. 24 No. 3 July 2020
Effect of administration of lead acetate

ASMAA S. BASSIT.et al sity. The diameter was measured by taking the largest and smaller

Statistical analysis of the data was done using SPSS software version 20.variable were represented by ( mean \pm standard deviation of the mean) independent ${ }_{-} t-$ test was used to compare the mean of the vari-able between different groups. Finally, the significance was considered according to the level of significance ( $\mathrm{P}$ value) as follows:

$\mathrm{P}>0.05 \quad$ (NS) $\rightarrow$ No significant difference.

$\mathrm{P} \leq 0.05(*) \rightarrow$ Significant difference.

$\mathrm{P} \leq 0.01 \quad(* *) \quad \rightarrow$ High significant difference.

$\mathrm{P} \leq 0.001(* * *) \rightarrow$ Very high significant difference.

\section{Result: \\ Group1: \\ One-week:}

During the first week, the ovary showed clear surface epithelium with well-appeared cortex and inner medullary region crowded with primary follicles and primordial follicle present near-surface (figure1). primordial follicle surrounded by one layer of squamous epithelium and primary follicles surrounded by 2-3 layers of cuboidal granulosa cell and a layer of the theca is established to grow(figure2).

\section{Two- weak:}

The ovary showed normal covering epithettlium, cortex and medulla with many follicles including primordial, primary, secondary, preantral follicles (figure3) secondary follicle surrounded by multiple layers of granulosa cells, also showed preantral and antral follicle in which there were multiple cavities appeared within the granulosa cell The oocyte increased in size and became surrounded by a layer of zona pellucida secreted by granulosa cell (figure 4).

\section{Group2:}

One-week:

The Ovary of lead treated animals showed irregular surface with a reduction in its size, there was a decrease in the number of follicles compare to normal, vacuolated Primordial follicles, Primary follicles were shrunken and damage with destruction in surrounded granulosa cell (figure 5), also oocytes were not was irregularly destructed, with the appearance of cavities in surrounded cytoplasm, Zona pellucida around the oocyte was destructed in most of the follicles and theca cell was disarrangeement (figure 6).

\section{Two-weak :}

The ovary showed irregular detached surface epithelium and different type of destructed follicle with damage oocyte (figure 7), distorted primordial follicle with the vacuolated oocyte, shrunken primary follicle, secondary follicles show the irregular distribution of granulosa cells, vacuolated oocyte with damage zona pellucida and also tertiary follicles was destructed (figure 8).

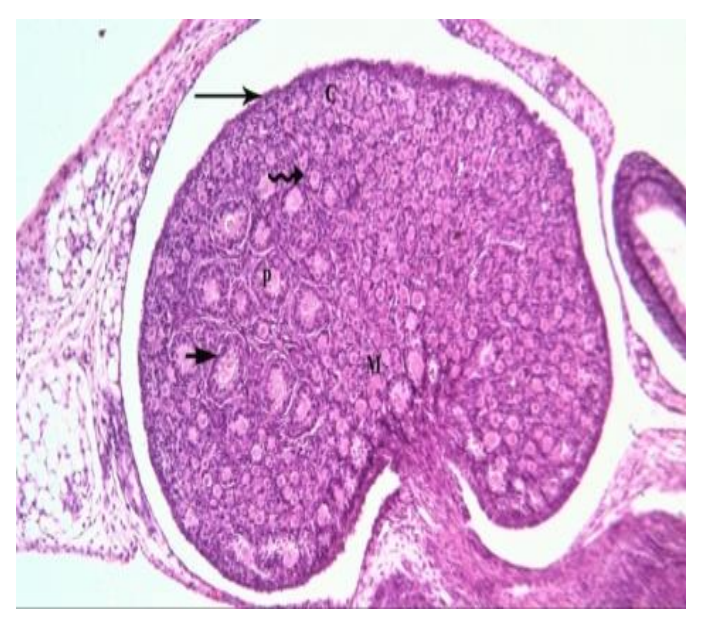

Figure1: photomicrograph section in the Ovary of control animals on PND 7 Control 
SOHAG MEDICAL JOURNAL

Vol. 24 No. 3 July 2020
Effect of administration of lead acetate

ASMAA S. BASSIT.et al ovary showing normal structure of surface epithelium (arrow), Cortex (C) and inner medullary (M), Primordial follicles (iregular arrow) mainly found at periphery. Primary follicles (P), (short arrow) are visible more at medulla.

H \& E.; X100.

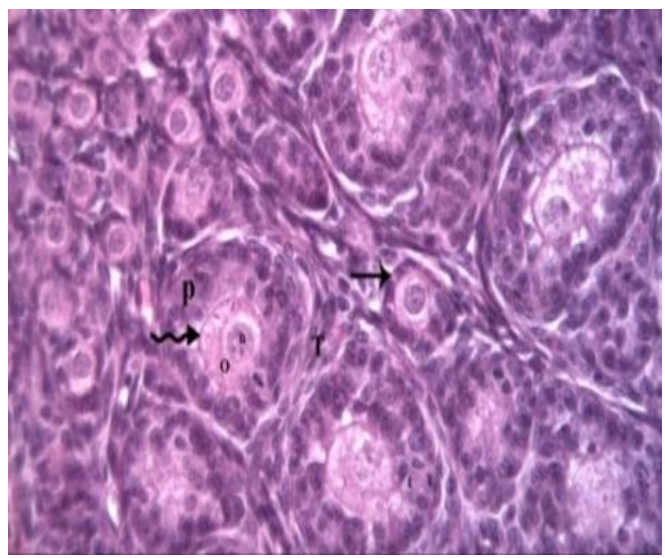

Figure 2: photomicrograph section in the Ovary of control animals on PND 7 Primordial follicles (arrow) with large eccentric nucleus mainly found at periphery. Primary follicles $(\mathbf{P})$ are visible having 2 layer of cuboidal follicular cells , oocyte (o) and nucleus (n) with zona pellucida (irregular arrow). Theca cells can also be identified (T).

H \& E.; X $\leqslant 00$.

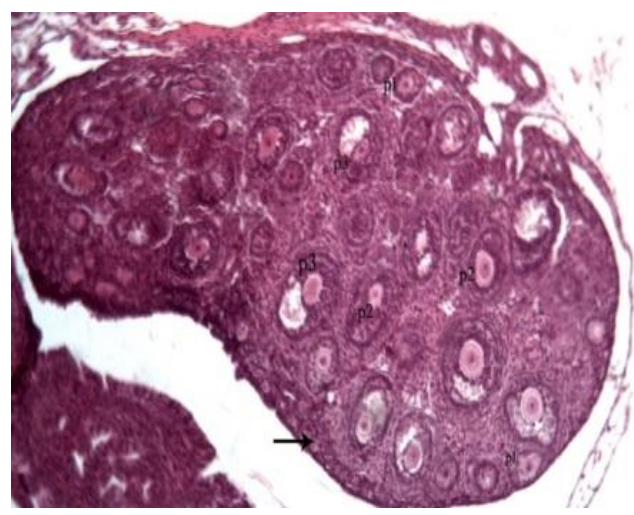

Figure 3: photomicrograph section in the Ovary of control animals on Control ovary on PND 14. showing normal primordial follicle (long arrow), Different types of developping follicles present in medulla, primary (P1) , secondary (p2), preantral follicle (p3).

H \& E.; X100.

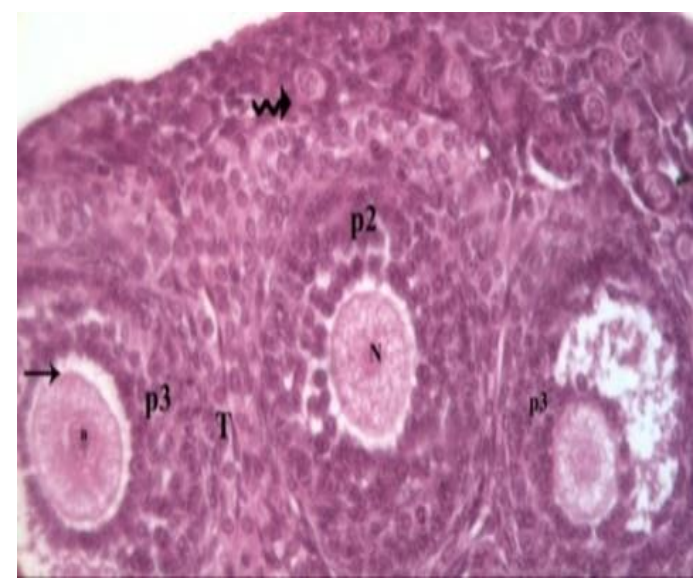

Figure 4 : photomicrograph section in the Ovary of control animals on Control ovary on PND 14. showing Different types of developping follicles primordial follicle (arrow) secondary (p2) contain oocyte with central nucleus, preantral follicle (P3) it contain primary oocyte with nucleus (n) surround with zona pellucid a (long arrow) with normal structure and surround with intact theca cell(T). (p3) another preantal follicle with appearance of fluid cavity.

H \& E.; X400.

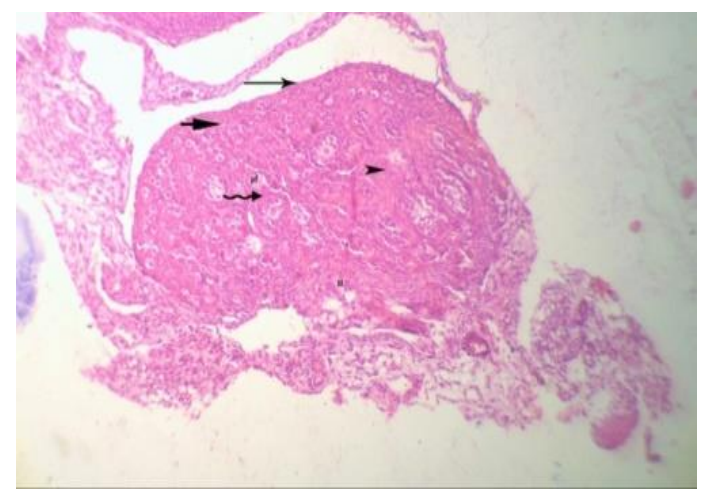

Figure 5: photomicrograph section in the Ovary of lead treated animals on PND 7, showing irregular surface of ovary with reduction in its size (long arrow), Primordial follicles (Pf) are reduced in size with irregular

distribution ,shrunken primary follicle (irregular arrow) Also there is area of fibrosis (head arrow), persistence of some 
SOHAG MEDICAL JOURNAL

Vol. 24 No. 3 July 2020

promidal germ cell indicate delay in development (short arrow).helium of ovary (H).

H \& E.; X100.

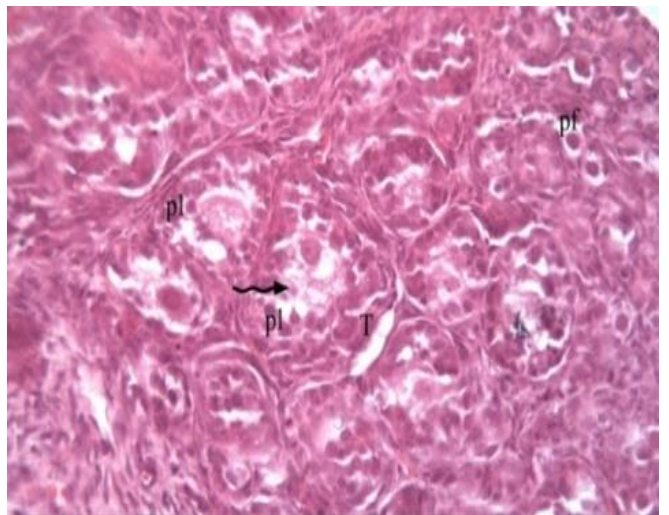

Figure 6: photomicrograph section in the Ovary of lead treated animals on PND 7, showing primordial follicle with vaculated oocyte (pf) ,multiple damaged primary follicle (p1), with shrunken oocyte and damage zona pellucida (irregular arrow) ,theca cell separated from surrounded cell (T).

H \& E.; X400.

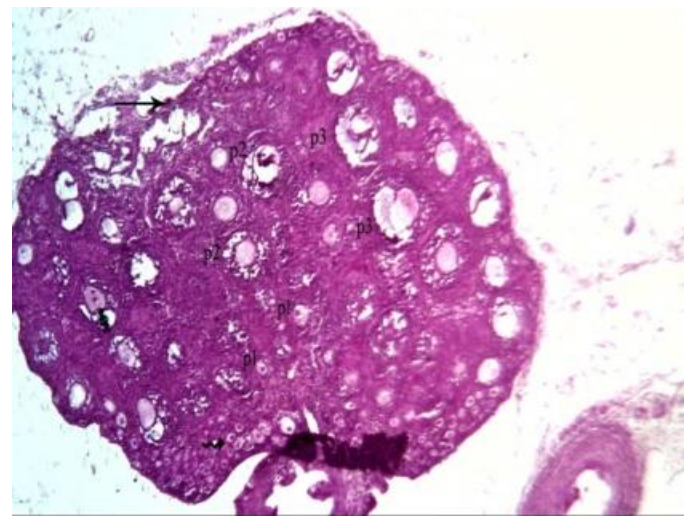

Figure 7: photomicrograph section in the Ovary of lead treated animals on PND 14, showing, irregular detached surface epithelium (arrow), primordial follicles vaculated (irregular arrow),primary follicle with shrunken nucleus (p1), secondary follicles( p2) with damage of granulosa cells ,destructed tertiary follicles (p3)with destructed oocytes and zona pellucida.

H \& E.; X100.

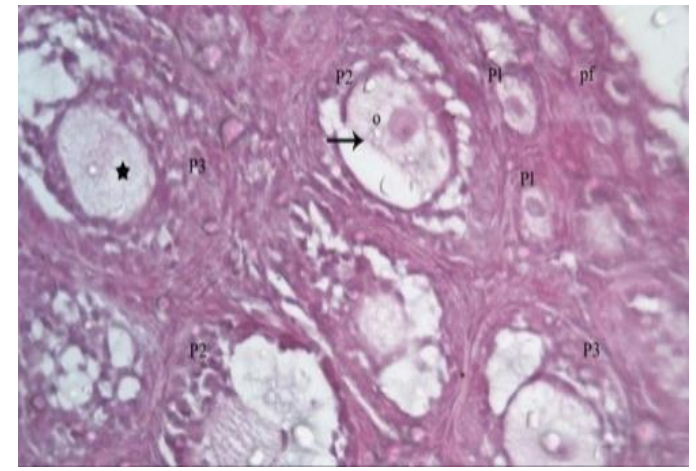

Figure 8: photomicrograph section in the Ovary of lead treated animals on PND 14, showing, multiple damaged follicle , distorted primordial follicle (pf), with vacuolated oocyte, shrunken primary follicle (p1), secondary follicles (p2) show irregular distribution of granulosa cells ,vacuolated oocyte (o)with damage zona pellucida (longarrow), ,destructed tertiary follicles (p3)with damage granulosa cell ,destructed oocytes $(*)$ without nucleus

\section{H \& E.; X400.}

Morphometric and statistical study :

The mean value of the diameter of the ovary in one weak group 2was (18.8) which highly significantly decrease $(\mathrm{P} \leq 0.001)$ compared with group 1 . (table1,histogram1).

The mean value of the diameter of the ovary in two weak group2 was (32.6), which is highly significantly decrease $(\mathrm{P} \leq 0.001)$ compared with the group1. (table1,histogram2).

\begin{tabular}{|l|l|l|}
\hline & control & treated \\
\hline 1 weak & $54.3 \pm 22.9$ & $18.8 \pm 1.45 * * *$ \\
\hline 2 weak & $46.8 \pm 4.8$ & $32.6 \pm 8.2 * * *$ \\
\hline
\end{tabular}

Table (1): showing Mean \pm SD diameter of ovary in control and lead-treated offspring Wistar rats at age of one and two weak . $\mathrm{P} \leq 0.001(* * *)$ :highly significant difference . 
SOHAG MEDICAL JOURNAL

Vol. 24 No. 3 July 2020

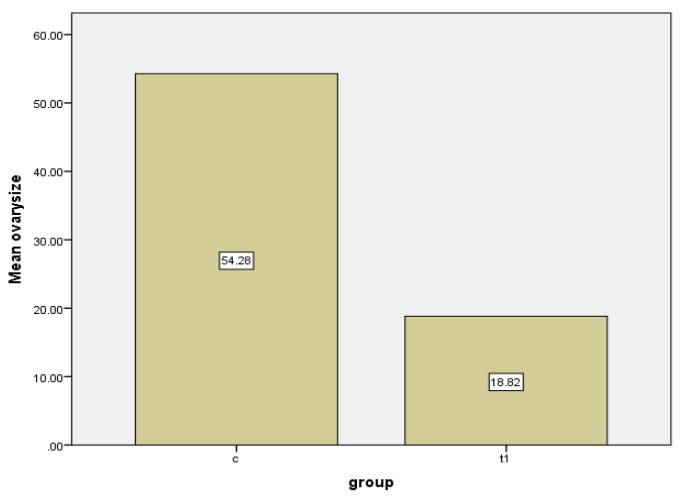

histogram (1): showing the diameter of ovary in $1 \mathrm{w}$ control and treated group.

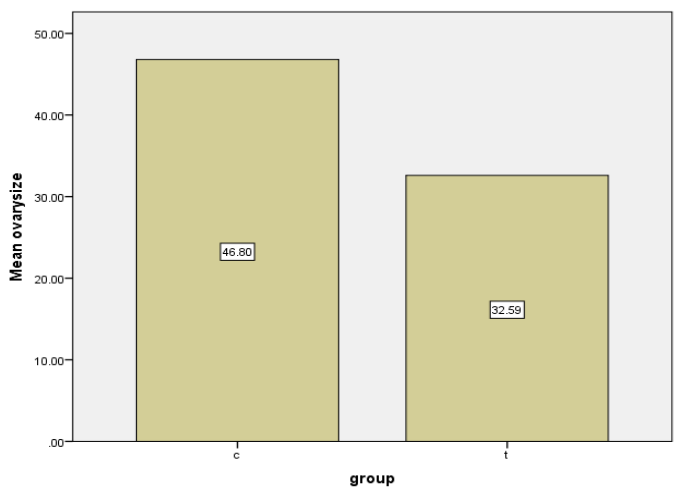

histogram (2): showing diameter of ovary in 2 weak control and treated group.

\section{DISCUSSION:}

An ovarian follicle is an important unit in the ovary. It contains the oocyte which undergoes ovulation, fertilization and forms an embryo. There is a similarity between the human and the rat ovary in shape, histological structure embryogenesis and physiology, but they differ in the size, the site and the onset of puberty. . (9)

Lead is one of the environmental pollutants, mainly found widely in industrial regions, The exposure to lead induces pathological changes A high level of also

lead in animals resulted in reproductive failure. (3).
The present study showed a decrease in the size of the ovary in lead treated group compares to normal, irregularity in the surface epithelium and affection of lead in a different type of follicle where it caused damage to its granulosa cell, shrunken to oocyte nucleus and destruction to zona pellucida.

This result agrees with Eugenia (10) who observed that Ovary following lead caused diffuse edemas, necrosis and damage to different ovarian follicles.

Sharma (8) who noticed that Lead affects the growth of primordial follicles during postnatal and juvenile life and causes damage in the structure of primary and secondary follicles with an increased number of atretic follicles.

Also Taupeau (11) illustrated that small doses of lead produce affection in foliculogenesis and lead to a decrease in the number of primordial, primary, secondary, antral follicles.

Junaid (12) also reported that lead treatment during pregnancy affected on fetus where cause delay transition from the primordial to the primary follicle stage so the number of primordial folicles in lead treated ovaries increase compare to control.

At the same time Shah ( 13) found that after intake of large doses of lead, decrease the number of ovarian follicles and increased atretic follicles.

And Naureen et (14) observed that intake of lead has effects on the histology of ovary where affect the process of follicle development.

\section{Conclusion:}

The administration to lead in the neonatal period affects the growth of primordial follicles during postnatal and juvenile life. 
SOHAG MEDICAL JOURNAL

Vol. 24 No. 3 July 2020
Effect of administration of lead acetate

ASMAA S. BASSIT.et al
Also, the effect on the growth of primary and secondary follicles and increased in the number of atretic follicles.

\section{References:}

1- Catherine A. Picut, Darlene Dixon, Michelle L. Simons, Donald G. Stump, George A. Parker, And amera K. Remick(2014): Postnatal Ovary Development in the Rat Morphologic Study and Correlation of Morphology to Neuroendocrine Parameters, Toxicologic Pathology, vol, XX, p4-6.

2- AnneN. Hirshfield and AndreaM. Desanti(1995): patterns of ovarian cell proliferation in rats during the embryonic period and the first 3 weeks postpartum, the biology of reproduction 53,p 12081221.

3- McDowell, L.R (2003): Minerals in Animal and Human Nutrition. 2nd ed. Elsevier Science, Amsterdam. 361-364.

4- Valdevane R Araujo, Melba O Gastal, Jose R Figueiredo and Eduardo L Gastal (2014): In-vitro culture of bovine preantral follicles, Reproductive Biology, and endocrinology vol.12.

5- Dietrich KN(1991): Human fetal lead exposure: Intrauterine growth, maturation and postnatal development. FundamApplToxicol; 16: 17 - 19.

6- Radostitis OM, Blood DC, Gay CC (1994). Veterinary medicine. A textbook of the diseases of cattle, sheep, pigs, goats and horses. Ed 8th, 31: 1469-1471.

7- Avazeri N, Denys A, Lefèvre B (2006): Lead cations to affect the control of both meiosis arrest and meiosis resumption of the mouse oocyte in vitro at least via the
PKC pathway Biochimie: 88(11): 18231829.

8- Sharma R, Panwar K, Barber I and Purohit A: Lead toxicity and postnatal development of Ovary. Int J Pharm Sci Res 2013; 4(4); 1575-1584.

9- Krishna, Amitabh \& Srivastava, Raj \& Banerjee, Arnab (2018): mammalian Ovary p1-16.

10- Eugenia Dumitrescu, Viorica Chiurciu, Florin Muslin, Roxana Popescu, Diana Brezovan5, Romeo T. Cristina (2015): Effects of long-term exposure of female rats to low levels of lead: ovary and uterus histological architecture change Turk J Biol 39: 284-289.

11- Taupe au C, Poupon J, Nome F and Lefevre B(2001): Lead accumulation in the mouse ovary after treatmentinduced follicular atresia. Reprod Toxicol; 15: 385-391.

12- Junaid M, Chowdhuri DK, Narayan R, Shanker R and Saxena DK (1997): Lead-induced changes in ovarian follicular development and maturation in mice. J Toxicol Environ Health; 50: $31-40$.

13- Shah AS, Shariff MM, Khan AS, Tayyab M, Chaudary AN, Ahmed N(2008): Correlation of blood lead levels with atresia of ovarian follicles of albino mice. Ann Pak Inst Med Sci 4: $188-192$.

14- Naureen Waseem, Shabnam Hamid, Shadab Ahmed Butt (2014): effect of lead acetate on the follicular count of mice ovary and the protective role of garlic extract, Pak Armed Forces Med J; 64 (1): 61-65. 